\title{
Philosophiques
}

\section{La causalité adéquate chez Spinoza}

\section{Lee C. Rice}

Volume 19, numéro 1, printemps 1992

URI : https://id.erudit.org/iderudit/027171ar

DOI : https://doi.org/10.7202/027171ar

Aller au sommaire du numéro

\section{Éditeur(s)}

Société de philosophie du Québec

\section{ISSN}

0316-2923 (imprimé)

1492-1391 (numérique)

Découvrir la revue

\section{Citer cet article}

Rice, L. C. (1992). La causalité adéquate chez Spinoza. Philosophiques, 19(1), 45-49. https://doi.org/10.7202/027171ar

\section{Résumé de l'article}

L'objectif de cet article est de proposer une analyse de deux conceptions distinctes de la causalité chez Spinoza. Selon la première (" émanative ») la nature-dieu serait la cause directe de toute action qui a lieu au niveau des choses finies; tandis que, selon la seconde (" consécutive ") toute action finie ferait partie d'une chaîne infinie de causes (dont chacune est toutefois finie) qui est répandue dans la durée. Je montre que cette causalité-ci n'est ni illusoire ni simplement derivative, contre les suggestions de plusieurs travaux récents sur Spinoza. En deuxième lieu nous proposons un modèle pour la causalité consécutive qui assure la possibilité que les êtres finis puissent fonctionner comme des causes adéquates au sens spinoziste dans le champ physique. Ici l'analyse s'oppose à plusieurs chercheurs qui soutiennent qu'il existe une rupture entre les attributs de la pensée et de l'étendue dans le système spinoziste. 


\title{
LA CAUSALITÉ ADÉQUATE CHEZ SPINOZA
}

\author{
par Lee C. Rice
}

RÉsumé. L'objectif de cet article est de proposer une analyse de deux conceptions distinctes de la causalité chez Spinoza. Selon la première ( émanative ») la nature-dieu serait la cause directe de toute action qui a lieu au niveau des choses finies; tandis que, selon la seconde ("consécutive") toute action finie ferait partie d'une chaîne infinie de causes (dont chacune est toutefois finie) qui est répandue dans la durée. Je montre que cette causalité-ci n'est ni illusoire ni simplement dérivative, contre les suggestions de plusieurs travaux récents sur Spinoza. En deuxième lieu nous proposons un modèle pour la causalité consécutive qui assure la possibilité que les êtres finis puissent fonctionner comme des causes adéquates au sens spinoziste dans le champ physique. Ici l'analyse s'oppose à plusieurs chercheurs qui soutiennent qu'il existe une rupture entre les attributs de la pensée et de l'étendue dans le système spinoziste.

ABSTRACT. This study addresses the problem of analyzing two distinct conceptions of causality in Spinoza. According to the first ("emanative») god-nature is the direct cause of every action which occurs at the level of finite things; while, according to the second ("sequential»), every finite action would be part of an infinite chain of causes (each finite) extended into duration. I show that sequential causality is neither illusory nor simply derivative, contrary to the suggestions made by several recent studies of Spinoza. Secondly, I offer a model for sequential causality which assures the possibility that finite beings may function as adequate causes (in Spinoza's sense) within the physical field. Here my analysis is opposed to those commentators who argue for a rupture in Spinoza between the attributes of thought and extension. 
En E1P18 Spinoza constate que «Dieu est la cause immanente de toutes choses et non pas cause transitive. "Dans le Court Traite $^{2}$ on lit que la cause immanente, dite aussi cause interne, ne produit rien en dehors d'elle puisque il $n^{\prime} y$ a absolument rien qui existe en dehors du Dieu-substance spinoziste. Cette espèce de causalité définit une chaîne finie de causes, dont chacune est à la fois infinie et éternelle ${ }^{3}$, ce qui la distingue d'une causalité temporelle et infinie prise en un sens consécutif:

Une chose singulière, ou, en d'autres termes, une chose quelconque qui est finie et dont l'existence est déterminée, ne peut exister ni être déterminée à agir, qu'elle ne soit déterminée à l'existence et à l'action par une autre cause, qui est également finie et dont l'existence est déterminée; et, à son tour, cette cause ne peut pas non plus exister ni être déterminée à agir, qu'une autre cause également finie et dont l'existence est déterminée, ne la détermine à l'existence et à l'action, et ainsi de suite à l'infini. (E1P28)

On peut par conséquent parler d'un modèle émanatif et $d^{\prime} u n$ second modèle consécutif chez Spinoza. Un problème central dont s'occupent plusieurs travaux récents sur Spinoza tient à la confrontation ou à la réconciliation de ces deux modèles. Une telle réconciliation devrait non seulement expliquer la juxtaposition presque constante des deux modèles à travers la première partie de l'Éthique ${ }^{5}$, mais, et cela est tout aussi important, il faut qu'elle jette une certaine lumière sur l'exposition que fait Spinoza de la causalité humaine, en particulier les remarques sur la causalité adéquate et inadéquate au début de la troisième partie.

Les analyses suivantes veulent démontrer que les solutions provisoires données jusqu'ici ont trop ignoré leurs implications (en vérité leurs contresens) pour la psychologie spinoziste. Je suggérerai en effet qu'il vaudrait mieux s'appuyer plus sur la psychologie (soit l'éthique) spinoziste tout en essayant d'esquisser une réconciliation générale de ces deux modèles.

\section{Le temps et l'illusion}

L'analyse "classique" du problème de la causalité chez Spinoza se trouve dans les œuvres de H. H. Joachim, qui critique Spinoza à partir d'une perspective idéaliste. À son avis, la logique spinoziste exige que le modèle émanatif soit le principal (même le seul), et que le second se réduise à une métaphore. L'erreur de Spinoza, d'après Joachim, fut de ne pas avoir vu que les modes finis, y compris leurs réactions, 
sont tout à fait illusoires ${ }^{6}$. L'interprétation idéaliste de Joachim a été vigoureusement rejetée par $\mathrm{H}$. F. Hallett ${ }^{7}$ qui a essayé de montrer que la causalité temporelle s'est introduite dans le système de Spinoza précisément pour éviter les conséquences idéalistes (et, d'après Hallett, absurdes) d'un immanentisme total. Hallett a été suivi par la plupart de commentateurs de nos jours ${ }^{8}$. Par opposition à toute vision idéaliste du monde, le "naturalisme » de Spinoza ${ }^{9}$ exige en effet que les deux modèles restent chacun efficace dans le système, et que chacun fournisse un aspect irréducible de toute explication dite causale.

L'analyse de Watt reste, je crois, le plus intégral des efforts pour clarifier la distinction entre les deux espèces de causalité. Pour lui, les deux modèles correspondent à deux effets différents de la causalité même. L'aspect émanatif de la causalité divine est responsable pour les essences de choses finies, tandis que l'aspect consécutif produit les existences dans la durée. Pour cette interprétation, E1P21-P23 sont en effet invoquées comme affirmant que les essences, et non pas l'existence des modes, doivent suivre directement de l'essence divine ${ }^{10}$. Si l'on soutient, comme insiste Watt lui-même, que ces essences jouissent d'une existence propre et tout à fait séparée de la durée où se trouvent les choses singulières dont elles sont les essences véritables, on fait de Spinoza un cartésien, justement comme le fit Gueroult dans son analyse des attributs ${ }^{11}$. On peut très bien échapper à une telle lecture, car tant s'en faut que le spinozisme soit réduit à une espèce faible du dualisme cartésien: le temps et l'éternité ne sont pas des régions en opposition mutuelle, mais ils restent plutôt deux aspects d'une seule réalité, celle de la nature ou de la substance.

Dans sa critique de Watt, Lennox juge qu'il y a des difficultés insurmontables pour toute interprétation qui, comme la sienne, sépare la causalité d'essence de la causalité d'existence entre les choses finies. Même nettoyée de son dualisme essentialiste, nous dit Lennox, Watt se trouve en conflit avec la définition de l'essence que donne Spinoza en E2Déf2:

Je dis qu'appartient à l'essence d'une chose cela qui, étant donné, fait que la chose est nécessairement posée, et qui, étant supprimée, fait que la chose est nécessairement supprimée; ou ce sans quoi la chose, et inversement ce qui sans la chose, ne peut ni être ni être conçu.

Selon l'interprétation de Lennox, cette définition identifie l'essence avec la cause de l'existence même des choses finies, 
ce qui mettrait en question la distinction que fait Watt entre les deux pour sauvegarder l'indépendance des deux modèles. Mais, contre Lennox, faut-il vraiment identifier le "tollitur" et le "ponitur" de la définition ${ }^{12}$ avec la cause de l'existence ou de la non-existence? Ces termes s'utilisent souvent, au XVIIe siècle comme au vingtième, en un sens logique ou explicatif: on "pose" (ponit) une chose pour considérer la nature des effets qu'elle produirait dans le cas où elle existerait en fait ${ }^{13}$. Les choses particulières, enfin, sont caractérisées moins par l'une ou l'autre propriété particulière de leur essence, que par les conditions dans lesquelles elles existent; c'est tout simplement ce que constate E1P28, comme nous venons de le voir ${ }^{14}$. Cette interprétation évite le dualisme caché d'un monde d'existence (la durée) qui dépend pour son intelligibilité d'un monde séparé et distinct d'essences primordiales. En critiquant le dualisme implicite de Watt, Lennox semble en effet tomber dans un piège leibnizien, où chaque essence "achève " son existence en agissant d'une manière indépendante de l'ordre temporel. Je crois plutôt que Martha Kneale a raison en suggérant que les similarités alléguées entre Spinoza et Leibniz - similarités dont se sert Lennox dans sa critique de Watt - sont plus imaginées que réelles ${ }^{15}$.

Une conséquence de ces réflexions serait l'acceptation provisoire de l'interprétation générale proposée par Watt. On ajoutera que cette interprétation $n^{\prime}$ est pas tout à fait différente de celle proposée par Curley pour résoudre le même ensemble de problèmes ${ }^{16}$. Curley réunit plusieurs preuves pour justifier sa thèse que la causalité émanative chez Spinoza porte en fin de compte sur l'explication nomologique de la science en général. Pour lui, le Dieu-substance est la cause des événements particuliers en tant qu'il forme l'ensemble des lois nécessaires de la nature en conformité avec lesquelles ces événements ont lieu; les modes individus (c'est-à-dire les individus finis) en seraient les causes en tant qu'ils agissent toujours selon ces lois éternelles du Dieu-substance:

On this interpretation, Spinoza's thesis that every infinite and eternal mode of the attribute of extension follows either directly from the absolute nature of the attribute of extension or indirectly from some other infinite mode which follows from the nature of extension (E1P23) - put in logical terms - amounts to the thesis that every scientific law relating to extended objects can be derived either directly from the fundamental laws governing extended objects or from a series of nomological propositions which terminate ultimately in the fundamental laws ${ }^{17}$. 
Un tel propos, d'après Lennox, conduit à un résultat inacceptable, puisqu'il rend nécessaire chacun des niveaux de causalité, sans que ni l'un ni l'autre ne soit suffisant en l'absence de $l^{\prime}$ autre ${ }^{18}$. Mais, encore une fois, ne faut-il pas rejeter un tel argument comme distinctement non spinoziste? - car la notion de «séparation » des deux niveaux ne trouve aucune justification dans le texte de Spinoza. Penser le Dieu-substance sans la série de modes finis $n^{\prime}$ est pas plus possible que penser la série sans le Dieu-substance. Dans l'établissement de telle ou telle explication causale, le choix entre les deux niveaux importe moins que d'en arriver à distinguer la contribution de chacun.

Vue dans cette perspective, l'analyse de Curley ne se différencie pas tellement en fin de compte de celle de Watt, bien qu'elle insiste sur des éléments différents. Les essences et l'existence des modes finis sont en effet inséparables; mais on peut fort bien distinguer les liens de causalité selon leurs aspects existentiel et essentiel. Spinoza nous en donne un exemple dans le scolie de E1P17:

Par exemple un homme est cause de l'existence d'un autre homme, mais non pas de son essence; car celle-ci est une vérité éternelle; ils peuvent donc, quant à l'essence, avoir quelque chose de commun, mais ils doivent être différents quant à l'existence. Par suite, si l'un cesse d'exister, l'autre n'en périra pas pour autant, mais si l'essence de l'un pouvait être détruite et devenir fausse, l'essence de l'autre serait également détruite.

On a rendu «prorsus convenire possunt » par "pouvoir avoir quelque chose de commun". Il faut pourtant souligner que Spinoza ne dit point que tout homme " partage » une essence commune avec tout autre; ce qui serait la position de Diane Steinberg ${ }^{19}$. Il vaut mieux suivre Matson en concevant l'essence individuelle (humaine) comme une sorte de chiffre génétique. Il est possible (mais nullement nécessaire) d'attribuer la même essence à deux hommes (des jumeaux monozygotiques) en sorte que, si l'essence (le chiffre) de l'un n'était pas possible, $l^{\prime}$ essence de l'autre ne le serait pas non plus $^{20}$.

Curley et Watt voient tous deux dans l'analyse spinoziste de la causalité consécutive, une affirmation de la véritable causalité des choses particulières et finies. Cette position se différencie bien de toute analyse qui nierait la réalité ou l'importance de cette espèce de causalité, et se rapprocherait sous cet aspect de la conception que donne Geulinc ${ }^{21}$ du système spinoziste du monde. Une telle position est en partie soutenue 
par les remarques, quelque peu égarantes il est vrai, que fait Spinoza en distinguant le temps, la durée, et l'éternité. Pour lui le temps signifie toujours une partie mesurée et abstraite de la durée ${ }^{22}$, tandis que la durée provient de l'effort ou conatus par lequel chaque être fini prolonge son existence dans le champ physique ${ }^{23}$. Bien entendu, dans la mesure où la durée comprend une infinité actuelle de causes finies (le champ temporel), Spinoza parle souvent de l'ordre commun de la nature (par exemple en E2P45S), mais ce n'est pas cet ordre qui forme la base du savoir inadéquat, contrairement à ce que prétend Gilead ${ }^{24}$; c'est plutôt la finitude de l'esprit humain en face de cet ensemble infini qui en est la source. Par conséquent la durée n'est ni illusion ni source d'erreur chez Spinoza.

Il faut cependant distinguer deux acceptions du mot «durée ». En un sens, la durée s'identifie à l'éternité, ou du moins elle y est étroitement unie. On parle alors de la durée infinie ou plutôt indéfinie, qui est identique à la nature même de l'existence ${ }^{25}$, et qui appartient, comme elle, aux modes en leurs rapports avec les attributs infinis ${ }^{26}$. Mais Rivaud a tort en concluant

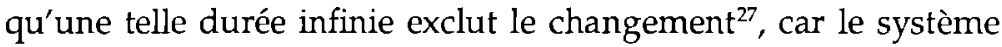
spinoziste embrasse un Dieu-substance qui consiste en une infinité de changements intérieurs sans aucun changement du point de vue de sa nature $\mathrm{mêm}^{28}$. La situation quotidienne $\mathrm{d}^{\prime}$ interaction entre les choses qui font le monde de notre expérience aurait, d'après mon analyse, un statut épistémologique différent de la Nature-Dieu eu égard aux explications causales dans le système spinoziste, mais cela ne déplace ni sa réalité ni son importance dans ce système. C'est en ce sens que Spinoza reste toujours fidèle à l'esprit de la nouvelle science galiléenne ${ }^{29}$.

\section{Causalité adéquate dans le champ physique}

Si ce que je viens d'exposer apparaît juste dans ses grandes lignes, on poursuivra en faisant remarquer contre Geulincx ou ceux qui veulent rapprocher la position de Spinoza de celle, par exemple, de Leibniz, que Spinoza insiste sur une causalité effective et temporelle pour les choses finies (les "modes finis " de son système). Insister et soutenir sont bien sûr des choses tout à fait différentes. Deux chercheurs, au moins, répondraient à l'analyse précédente que, malgré toute intention prétendue de la part de Spinoza, son analyse de la causalité rend impossible une vraie effectivité des modes finis, ou du moins toute effectivité qui soit autre que partielle ou 
fragmentée. La base d'une telle réponse se situe dans les deux premières définitions que nous donne Spinoza au début de la $3 e$ partie de l'Éthique:

Déf. I: J'appelle cause adéquate celle qui permet, par ellemême, de percevoir clairement et distinctement son effet, mais j'appelle cause inadéquate ou partielle celle qui ne permet pas de comprendre son effet par elle seule.

Déf. II: Je dis que nous agissons lorsqu'il se produit en nous ou hors de nous quelque chose dont nous sommes la cause adéquate $[\ldots]$

Le savoir adéquat serait par conséquent une connaissance dont l'esprit fini est la cause adéquate, car autrement il s'agirait d'un savoir inadéquat (Spinoza parle plutôt d'une idée inadéquate).

Mais une telle causalité serait-elle même logiquement possible du côté d'un être fini ? Certains interprètes pensent que non, par exemple Diane Steinberg:

So far I have argued that (a) Spinoza's conception of the physical world is such that no extended thing can ever act in his technical sense; while (b) his conception of adequate knowledge implies that any mind which has such knowledge or engages in adequate thinking, to that extent, acts. Thus, (c) on his theory of the mind as the idea of (corresponding to) an actually existing body, Spinoza is unable to give an account of the ability of the mind to engage in adequate thinking, for there can be no physical process which corresponds to the mental function of adequate thought ${ }^{30}$.

J'ai soutenu ailleurs que l'analyse qu'esquisse Steinberg en ce qui concerne l'éternité de l'esprit n'est pas acceptable comme interprétation de la $5 e$ partie de l'Éthique ${ }^{31}$, mais cet aspect de son analyse ne nous intéresse pas ici. Tournonsnous plutôt vers la critique (impliquée) de la causalité consécutive.

Le support textuel qu'offre l'analyse de Steinberg nous renvoie à E1P28, le passage examiné tout à l'heure où Spinoza parle d'une chaîne infinie de causes consécutives répandue dans la durée. Steinberg (pp. 45-46) nous invite à considérer une balle $A$ qui est frappée par une autre balle $B$ qui lui communique du mouvement. Mais, insiste-t-elle, l'action de $B$ n'est point la cause adéquate du mouvement de A; car, pour comprendre clairement et distinctement le mouvement de $A$, il nous faudrait aussi comprendre comment s'est initié le mouvement en $B$, et pour comprendre le mouvement d'une balle $C$ qui frappe $B$, il nous faudrait également comprendre 
l'origine du mouvement de C, etc., etc. Contre Steinberg, on peut alors se demander où Spinoza nous dit que, pour comprendre un effet, il faut aussi comprendre toutes les causes de ses causes? De même on se demandera où donc Spinoza nous dit qu'une cause partielle est, par définition, une cause dont la causalité même est causée? Le physicien, en particulier le physicien spinoziste ${ }^{32}$, insisterait sur le fait qu'on peut bien comprendre le mouvement de A pourvu qu'on comprenne bien la façon dont ce mouvement s'initie par l'action de $B$ sur $\mathrm{A}$, et cela sans avoir à connaître l'origine précise de cette deuxième action. Car la première explication (pourquoi et comment le mouvement s'est initié) constitue le paradigme même de la physique spinoziste, y compris les lois premières du mouvement ${ }^{33}$.

Prenons un autre exemple, suivant la forme générale que nous propose Steinberg. Considérons cette fois deux balles, B et $C$, toutes les deux en mouvement, qui frappent simultanément A, lui communiquant un mouvement qui est la résultante de deux vecteurs. Il s'agit ici de la loi générale du parallélogramme, déduite des lois encore plus générales par Spinoza dans son commentaire sur Descartes ${ }^{34}$. Selon E3Déf2 chacune des balles $B$ et $C$ serait une cause inadéquate du mouvement de $A-$ non point parce que leurs mouvements ont des causes antérieures, mais plutôt parce que chacune ne contribue qu'à une partie de la quantité et de la direction du mouvement que reçoit $A$. Cela ne nous donne-t-il pas exactement enfin le sens qu'exige Spinoza pour la cause "partielle »? Bref, nous avons une série de causes, faite de causes partielles ou inadéquates dont chacune agit simultanément pour produire un seul effet; mais, d'un autre côté, une série dont les causes sont dispersées dans la durée (chacune agissant sur sa prochaine) peut bien contenir des causes adéquates. Je dis "peut " puisque les deux séries peuvent bien se trouver dans le même champ d'activité. Il n'y a rien ici d'étonnant: les deux structures supportant de tels liens de causalité sont reconnues et comprises par la physique cartésienne, et également par la science moderne.

$\mathrm{Au}$ lieu de soutenir que toute la causalité physique finie serait inadéquate, comme le fait Steinberg dans son analyse, mon interprétation voudrait suggérer au contraire que toute action de la part d'un corps quelconque serait adéquate à tel ou tel effet. Considérons par exemple n'importe quel effet (activité). Même si sa cause était un ensemble complexe de choses, chacune agissant simultanément avec les autres, ne 
pourrait-on pas avancer que chacune de ses causes "partielles " serait exclusivement responsable d'une partie de l'effet total, et donc qu'elle serait la cause adéquate de cette partie? Pour soutenir une telle conséquence peut-être faudrait-il faire une analyse de l'individuation (des effets et des causes) plus précise que celle que je donne ici. Je dirais intuitivement que cela n'est pas nécessaire, mais il faut avouer que la tâche est lourde si l'on veut entreprendre une explication raisonnablement complète de l'individuation chez Spinoza.

Si l'on oublie cette explication de la causalité consécutive et adéquate, et si on veut tout de même éviter de tomber dans celle du parallélisme implicite à la position de Steinberg, on pourrait alors adopter une version un peu affaiblie de son analyse, selon laquelle la causalité des choses finies ne peut être adéquate que dans une perspective excluant la durée. $C^{\prime}$ est une telle solution que nous propose Gilead:

A "responsible" particular thing which is a real cause is one whose effect can be clearly and distinctly perceived by means of that cause solely [...] However, man perceived as a link in the transient "chain", is not "responsible" for his actions and is only an impotent servant of fate, chance and arbitrary circumstances; whereas conceived qua link in the immanent causal chain he is responsible for his part, is free and active, and his real power, finite as it is, is fully expressed in its effect on the whole of Reality. As a free agent he does not act in place and time $e^{35}$.

Gilead ne nous explique pas comment un sujet peut vraiment agir sans à la fois agir dans l'espace et dans la durée; mais, parce qu'il prétend qu'une telle action n'est pas exclusivement mentale, sa position est différente de celle de Steinberg bien $q^{\prime}$ 'il s'en défende ${ }^{36}$. Steinberg prétend en effet qu'un être fini ne peut jamais fonctionner comme cause adéquate, au moins dans le champ physique ${ }^{37}$, et Gilead s'inscrit en faux contre une telle thèse ${ }^{38}$. Si la distinction que j'ai faite entre les liens simultanés et temporels suffit comme exemple de ce que propose Spinoza en E3Déf1, il ne semble pas alors nécessaire de chercher un sens " essentiel » (ou hors-la-durée) pour la causalité adéquate, comme le fait Gilead.

Mon interprétation soulève cependant plusieurs problèmes dont il nous faut maintenant faire mention. D'abord, la possibilité de la causalité adéquate dans le sens où nous l'avons développée dépend d'une distinction antérieure et systématique entre une cause et une condition pour un effet quelconque. Car la notion 
de champ physique présuppose que toute action ait lieu précisément à cause du milieu physique total qui lui est voisin. Si l'on identifie ce milieu ( $c$ 'est-à-dire les conditions qui rendent possible la causalité productrice) avec l'ensemble des causes de n'importe quel effet qui $s^{\prime} y$ produrait, il s'ensuit qu'il $n^{\prime} y$ aura jamais d'autre cause adéquate que la nature tout entière. Mais fautil adopter une telle position en regard de la distinction (en l'absence d'une justification logique pour une telle distinction) entre les conditions et les causes? Il me semble que non, au moins dans une perspective spinoziste. Certes, il n'y a rien dans le texte de Spinoza qui suggère qu'il envisageait cette distinction. Mais ce n'est pas conclure que la distinction est tout à fait claire dans son système, ou même soutenable. La distinction était implicite dans la science que connaissait Spinoza, et je crois bien qu'elle l'est aussi dans la nôtre. Tout cela nous donne sa position historique. Pour sa justification logique, il faudrait considérer le système scientifique dont s'est servi Spinoza pour son modèle principal.

Ce qui importe, c'est que les catégories et les structures explicatives dont se sert Spinoza sont des parties intégrantes de son "matérialisme naturaliste ${ }^{39}$, et que le système spinoziste n'est pas idéaliste ou transcendentaliste dans le sens où ont voulu l'entendre ces chercheurs qui trouvent dans les remarques de Spinoza sur la causalité, un effort pour soustraire le monde ordinaire physique de toute effectivité ou encore à l'ordre de l'explication scientifique. Du point de vue de son réalisme scientifique, Spinoza laisse ce monde exactement comme il le trouva. Sa quête philosophique ne consiste pas à en trouver un autre, mais plutôt à revêtir ce monde (le seul qui existe) d'une intelligibilité raisonnée.

Département of Philosophy

Marquette University, Milwaukee, Wisconsin

\section{NOTES}

1. Je voudrais exprimer ma reconnaissance aux lecteurs ainsi qu'au rédacteur de Philosophiques pour leurs critiques et leurs suggestions, et aussi à Étienne Barbone, Dominique Vandevelde, et Pol Vandevelde (Marquette University) pour leur assistance dans la préparation de la version finale du manuscrit. C'est moi qui reste seul blâmable pour ses fautes, soit de langue soit de raisonne- 
ment. Pour le texte latin de Spinoza, je préfère l'édition des CEuvres Complètes par J. Van Vloten et J. P. N. Land [Benedicti de Spinoza opera quotquot reperta sunt, 3e édition. 4 tomes. La Haye: M. Nijhoff, 1914] à celle de Carl Gebhardt [Opera, im Auftrag der Heidelberger Akademie der Wissenschaften, 4 tomes. Heidelberg: Carl Winters Verlag, 1925]. Une nouvelle édition critique, en cours de rédaction en Hollande, ressemblera de près à celle de Van Vloten et Land. La traduction française utilisée est tirée de L'Éthique, introduction, traduction, notes et commentaire par Robert Misrahi (Paris: P.U.F., 1990). Toute référence à l'Éthique est interne. E2P13Cor est le corollaire à la proposition 13 de la 2 e partie, etc. Autres abréviations: Dém(onstration), Schol(ie), App(endice), et Déf(inition).

2. CT, I, Premier Dialogue. Voir la traduction de Madeleine Francès dans l'édition de La Pléiade (Paris: Gallimard, 1954) des CEuvres Complètes, et aussi Gilbert Boss, L'enseignement de Spinoza: Commentaire du "Court Traité », (Zurich: Grand Midi, 1982), 33-35.

3. E1P21: "Tout ce qui suit de la nature absolue d'un attribut de Dieu a dû exister de tout temps et être infini, c'est-à-dire est éternel et infini par cet attribut."

4. Voir Étienne Balibar, “ Causalité, individualité, substance: réflexions sur l'ontologie de Spinoza », Spinoza: Issues and Directions, éd. par E. Curley et P.-F. Moreau (Leiden: E. J. Brill, 1990), 58-76; le deuxième chapitre de E. M. Curley, Spinoza's Metaphysics: An Essay in Interpretation, (Cambridge: Harvard UP, 1969), en particulier 55-67; Henri Daudin, "Sur quelques points de la philosophie de Spinoza", Revue de Métaphysique et de Morale, 57 (1952), 313-324; T. M. Forsyth, "Spinoza's Doctrine of God in Relation to his Conception of Causality ", Philosophy, 23, 291-301 [réimpression dans Studies in Spinoza, éd. Paul Kashap (Berkeley: Univ. of California Press, 1972), 3-15]; Amihud Gilead, "Spinoza's Two Causal Chains ", Kant Studien, 81 (1990), 454- 475; R. Henrard, De spinozistische Achtergrond van de Beweging van Tachtig, (Leiden: E. J. Brill, 1974); James G. Lennox, "The Causality of Finite Modes in Spinoza's Ethics ", The Canadian Journal of Philosophy, VI (1976), 479-500; Hans-Christian Lucas, "Causa sive ratio ", Cahiers Spinoza, 4 (1983), 171-204; Lee Rice, "Spinoza on Individuation », The Monist, 55 (1971), 640-659; Yochanan Silman, "La causalité dans la philosophie de Spinoza ", Iyyun, 36 (1987), 3-12; Marx Wartofsky, "Action and Passion: Spinoza's Construction of a Scientific Psychology ", Spinoza, éd. par M. Grene (Garden City: Doubleday, 1973), 329-353; A. J. Watt, " The Causality of God in Spinoza's Philosophy ", The Canadian Journal of Philosophy, II (1972), 171-189; et Harold Zellner, "Spinoza's Causal Likeness Principle ", Philosophy Research Archives, 11 (1985), 453- 462.

5. Voir Lennox, 484:

The problem we are facing, then, is such a serious one because, far from seeing these two accounts of God's causality of finite 
modes as incompatible, Spinoza makes persistent use of the distinction between them, as if it solved difficulties rather than created them.

6. Voir H. Joachim, A Study of the Ethics of Spinoza, (Oxford: Clarendon Press, 1901), 78: "In the timeless actuality of the modal system, in the completeness of "natura naturata", there is no individual "essentia" or "existentia" except that of the whole system."

7. H. F. Hallett, "Dr. Johnson's Refutation of Bishop Berkeley », Mind, 55 (1947), 132-147.

8. Voir Lennox, 485: «[...] Spinoza realizes that finite modes cannot follow from infinite substance. It is because he knows this that the serial mode of God's causality is introduced. "

9. Je me sers ici de la phrase de E. M. Curley, "Man and Nature in Spinoza », Spinoza's Philosophy of Man, éd. par Jon Wetlesen (Oslo: Universitetsforlaget, 1978), 19-26.

10. Voir Watt, 181-184.

11. Voir Martial Gueroult, Spinoza, I: Dieu, (Paris: Aubier-Montaigne, 1968), 265-268.

12. "Ad essentiam alicujus rei id pertinere dico, quo dato res necessario ponitur, et quo sublato res necessario tollitur... " Il s'agit ici de définir la phrase, "ad essentiam pertinere", et non pas l'essence tout simplement. Je m'accorde en général avec la conception causale de l'essence spinoziste proposée par Wallace Matson. Voir son "Body Essence and Mind Eternity", dans Spinoza: Issues and Directions, éd. par E. Curley et P.-F. Moreau (Leiden: E. J. Brill, 1990), 82-95.

13. Albert Rivaud, Les notions d'essence et d'existence dans la philosophie de Spinoza, (Paris: Alcan, 1906), 60-63.

14. Voir aussi E1P24: « Rerum a deo productarum essentia non involvit existentiam... ". Et aussi E1P24Dém: "Adeoque earum essentia neque suae existentiae neque suae durationis potest esse causa. "

15. Martha Kneale, "Leibniz and Spinoza on Activity », Leibniz: Critical Essays, éd. par H. Frankfurt (Garden City: Doubleday, 1972), 216217. Kneale a raison aussi, je crois, en suggérant que cette interprétation leibnizienne de Spinoza a ses origines dans l'exposé sur Leibniz par Bertrand Russell. Russell semble ignorer que Leibniz parle presque toujours d'états comme l'activité, mais Spinoza parle des événements comme l'action.

16. Ici je me trouve aussi en désaccord avec Lennox qui trouve Curley nettement opposé à Watt. La situation est cependant plus complexe que mon analyse ne le suggère; car l'analyse que propose Watt de l'individu ("res particularis») est tout à fait opposée à celle de Curley; mais j'insisterais ici sur le fait que c'est Curley qui a raison. Voir notre article, “Tanquam Exemplar Naturæ Humanæ: 
Spinoza on Human Nature, " à paraître dans The Modern Schoolman, et aussi Lee Rice, "Spinoza on Individuation".

17. E. Curley, Spinoza's Metaphysics: An Essay in Interpretation, 59.

18. Lennox, 487:

This interpretation, as plausible as it sounds, presents some serious difficulties. It postulates two sources of causality, each necessary, and jointly sufficient, for the determination of finite modes. Yet Spinoza is perfectly clear that the essence of God is necessary and sufficient for the production of all things (E1p16-18)..."

19. "Spinoza's Ethical Doctrine and the Unity of Human Nature ", Journal of the History of Philosophy, 22 (1984), 303-324.

20. Voir Wallace Matson, "Body Essence and Mind Eternity in Spinoza ", dans Spinoza: Issues and Directions, 82-95; et aussi le commentaire par Henry Allison (96-101).

21. Voir H. J. de Vleeschauwer, Three Centuries of Geulincx Research, (Pretoria: Communications of the University of South Africa, 1957), 48-50.

22. Voir C. L. Hardin, «Spinoza on Immortality and Time», Southwestern Journal of Philosophy, 8 (1977), 129-138 [réimpression dans Spinoza: New Perspectives, éd. par R. Shahan et J. Buro (Norman: University of Oklahoma Press, 1978].

23. Voir Lee Rice, "Emotion, Appetition, and Conatus in Spinoza ", Revue Internationale de Philosophie, 31 (1977), 101-116. La notion de " champ physique " est empruntée à Jonathan Bennett [A Study of Spinoza's Ethics, (Indianapolis: Hackett, 1984), 97-105].

24. Voir Amihud Gilead, "Spinoza's Two Causal Chains ", 462: "Indeed, for Spinoza, the common order of nature means inadequate cognition. »

25. E2Déf5: "La durée est la continuation indéfinie de l'existence. " E2P45schol:

Par existence je n'entends pas ici la durée, c'est-à-dire l'existence en tant qu'elle est conçue abstraitement et comme une certaine forme de la quantité. Car je parle de la nature même de l'existence, celle qui est attribuée aux choses singulières en raison du fait qu'une infinité de choses en une infinité de modes suivent de la nécessité éternelle de la nature de Dieu [...]

26. Voir la Lettre 12 à L. Meyer, 20 avril 1663, et aussi Albert Rivaud, Les notions d'essence et d'existence dans la philosophie de Spinoza, 120-124.

27. Rivaud, 123. L'identification, erronée à notre avis, de la durée et de l'éternité est proposée aussi par Martha Kneale, " Eternity and 
Sempiternity ", Spinoza, éd. par M. Grene (Garden City: Doubleday, 1973), 227-240.

28. E2P13Lemm7Schol:

Et si nous poursuivons ce raisonnement à l'infini, nous concevrons aisément que la nature entière est un individu unique, dont les parties, c'est-à-dire tous les corps, varient d'une infinité de façons, sans aucun changement de l'individu total. »

Pour un commentaire sur ce passage, voir Lee Rice, «Individuation in Spinoza ".

29. Nous sommes pour cette raison en désaccord avec Daniel Garber ["Spinoza's Worlds: Reflections on Balibar on Spinoza", dans Spinoza: Issues and Directions, éd. par E. Curley et P.-F. Moreau, 77-81], pour qui la métaphysique (et donc la physique) de Spinoza " ne décrit point le monde où nous vivons " [79]. Pour le modèle galiléen voir Lécrivain, 2, 99-118, et aussi David Savan, "Spinoza: Scientist and Theorist of Scientific Method ", dans Spinoza and the Sciences, éd. par M. Grene and D. Nails (Dordrect: Reidel, 1986), 95-124.

30. Diane Steinberg, "Spinoza's Theory of the Eternity of Mind", Canadian Journal of Philosophy, 11 (1981), 53.

31. Voir “ Tanquam Exemplar Naturae Humanae: Spinoza on Human Nature ».

32. Voir l'étude essentielle d'André Lécrivain, «Spinoza et la physique cartésienne ", Cahiers Spinoza, 1 (1977), 235-236; 2 (1978), 93-206. La discussion du cercle de mouvement [143-152] explicite clairement les présupposés de la pensée de Spinoza sur l'explication du mouvement dans le champ physique. Voir aussi les Principes de la philosophie de Descartes (PPC) de Spinoza pour une discussion du cercle de mouvement (PPC2P8 et P8cor).

33. Voir Harold Zellner, "Spinoza's Causal Likeness Principle » 459461; Henri Daudin, "Sur quelques points de la philosophie de Spinoza ", Revue de Métaphysique et de Morale, 57 (1952), 313-324; et Yochanan Silman, "La causalité dans la philosophie de Spinoza ", Iyyun, 36 (1987), 3-12. Au lieu des mots "émanatif" et "consécutif", Daudin emploie "mathémathique" et "mécanique". J'ai préféré ceux-là parce que, chez Spinoza, la causalité "mécanique " s'explique par des lois tout à fait mathématisées. Silman souligne, comme Lécrivain, le sens où la pluralité même se lève dans le champ physique comme résultat de cette causalité (ce qui est équivalent à ce qu'on appelle de nos jours les lois du choc).

34. Voir PPC2P27, et le commentaire par Lécrivain, Cahiers Spinoza, $2,184-187$

35. Amihud Gilead, "Spinoza's Two Causal Chains ", 464-465.

36. Gilead, 469-470, ff. 108. 
37. Steinberg concède que cette interprétation rompt le parallélisme développé par Spinoza à partir de E2P7, mais elle semble croire qu'il faille payer une telle rupture pour en arriver à une analyse consistante de la $5 e$ partie de l'Éthique.

38. Voir aussi Amihud Gilead, "Spinoza's principium individuationis and Personal Identity ", International Studies in Philosophy, 15 (1983), 41-57. Gilead constate (50-51) que les choses finies sont des causes adéquates quelquefois, et il n'y a aucune indication dans l'article plus récent qu'il ait changé d'avis sur ce sujet.

39. Le sens exact de cette phrase est expliqué dans l'étude excellente d'Émilia Giancotti Boscherini, "Man as a Part of Nature », Spinoza's Philosophy of Man, éd. par Jon Wetlesen, 85-96. 